www. revistadyo.com

\title{
Estrategias de Offshoring - Reshoring (2015-2018) desde un enfoque de análisis de red
}

Justo de-Jorge Moreno, Roberto Marín Rey y Fernando Merino de Lucas

https://doi.org/10.37610/dyo.v0i73.592
Recibido: 12 de Octubre de 2020

Aceptado: 23 de Diciembre de 2020

\section{Resumen}

El principal objetivo de este trabajo utilizando la información proporcionada por el European Reshoring Monitoring en el periodo 2015-2018, es determinar la importancia que cada país tiene, en las decisiones estratégicas de offshoring y Reshoring llevadas a cabo por las empresas, ante la búsqueda de ventajas competitivas en los procesos de búsquedas de nuevas ubicaciones para toda (o parte) de su cadena de valor. Los principales resultados revelan que no sólo países emergentes como China fueron el destino de la oleada de offshoring, sino que también países avanzados como Alemania o Italia fueron el destino de estos cambios en la localización de la producción. La oleada de segundos cambios en la localización, teniendo a China como epicentro, fue hacia Reino Unido, Francia, Italia, o países escandinavos entre otros. En esta relocalización, las empresas analizadas no buscaban explotar ventajas adicionales de costes, sino el retorno a países avanzados motivada por una pluralidad de factores influyentes que recoge la literatura, como la imagen de país entre otros. Finalmente, los resultados han puesto de manifiesto que el backshoring es más común entre empresas francesas, británicas e italianas.

\section{Palabras clave}

Deslocalización; Redeslocalización; Análisis de red, cadena de valor.

\section{Introducción}

A lo largo de los últimos decenios del siglo XX, las empresas de los países avanzados desarrollaron una estrategia masiva para optimizar sus procesos de producción, buscando el modo de suministro óptimo para cada una de las etapas, así como la ubicación de dichas actividades. Después de esta primera ola y ahora en el siglo XXI, una segunda ola o reubicación está ocurriendo. Esta segunda ola supone un replanteamiento de la optimización de los lugares en los que la deslocalización implantó las actividades que tradicionalmente habían sido acogidas en las economías avanzadas, de la Conferencia de las Naciones Unidas sobre Comercio y Desarrollo (en adelante, UNCTAD; 2013). Aunque no todas las deslocalizaciones implicaron Inversión Extranjera Directa (en adelante IED) (sólo lo que se denomina "deslocalización

\footnotetext{
Justo de-Jorge Moreno * justo.dejorge@uah.es

iD ORCID: 0000-0002-8326-3046

Roberto Marín Rey*

rober.henares@gmail.com

Fernando Merino de Lucas *** fmerino@um.es

* Dpto. de Economía y Dirección de Empresas Facultad de Ciencias Económicas, Empresariales y Turismo.Plaza de la Victoria 2 Alcalá de Henares 28802 Universidad de Alcalá ** Facultad de Economía y Empresa Campus de Espinardo E-30100 Murcia (Spain) Universidad de Murcia
}

cautiva" y no la externalización al extranjero), los datos de la UNCTAD indican que la relación entre las desinversiones y las salidas brutas de capital va desde alrededor del 30\% en el Reino Unido y Japón hasta el $64,1 \%$ en Alemania en el período 2000-2010.

Como revelaron las informaciones anecdóticas que aparecieron en las noticias o las corrientes de IED, se empezó a prestar algún tipo de atención pública a las empresas que volvían a casa después de haber desplazado su producción al extranjero. Recuerdan una atención especial, ya que en segmentos importantes de las economías avanzadas, el temor a la deslocalización se consideraba una amenaza (véase Garner, 2004, Head y otros, 2009) en muchos aspectos, pero especialmente para el empleo (Boehm y otros, 2019), pero también para la recaudación de impuestos o el potencial innovador de un país, ya que se consideraba importante la proximidad geográfica entre la producción y la investigación, al tiempo que se descuidaban otros efectos positivos (véase Carluccio y otros, 2019, Amiti y Wei, 2009, etc.). La resonancia de algunos de esos casos se incrementó en medio de las campañas nacionales de compra en algunas economías clave (como la de los Estados Unidos o el Reino Unido).

Un examen más detallado de esos casos reveló que las empresas estaban reconsiderando muchas de sus decisiones anteriores. Algunos autores señalan que más que un simple replanteamiento de lo que está sucediendo es la necesidad de llegar a la orilla derecha (digamos que para encontrar el lugar adecuado teniendo en cuenta que el entorno es volátil 
y que las necesidades de las empresas cambian con el tiempo) y que la respuesta dista mucho de ser estática (Tate, Bals, 2017). En esta tendencia de las empresas a buscar nuevas localizaciones se observaron diferentes opciones. En algunos casos, las empresas trasladan las actividades a nuevos lugares, en otros casos volvían a su lugar de origen, en otros las trasladan a países vecinos al domicilio de la empresa. Así pues, esta variedad de casos, la necesidad de conocer las razones, las diferencias entre las actividades, planteó interesantes retos de investigación (Ellram y otros, 2013, Tate, 2014).

La literatura se apartó inicialmente del mismo marco que analizaba la deslocalización para explicar estos segundos cambios combinando los resultados de los negocios internacionales y la gestión de la cadena de suministro. Como marco teórico, demostró ser fructífero, y su utilización para estos nuevos cambios ayudó a comprender este nuevo fenómeno (véase un buen resumen en Benstead et al. 2017). Sin embargo, en la bibliografía empírica se observó que no todos los factores eran igualmente importantes y que los factores que se consideraban menos importantes para la deslocalización emergen como críticos en la decisión de regresar al país. En este sentido, podemos decir que los estudios empíricos estaban creando la base para comprender plenamente el proceso de deslocalización y sus peculiaridades.

Sin embargo, la literatura empírica se enfrentó a importantes limitaciones para hacer esas contribuciones. Por una parte, las cifras de comercio internacional/IDE no informan de las deslocalizaciones (como no lo hacen con la deslocalización) o lo hacen a un nivel tan agregado que no es posible obtener una visión de la empresa a partir de ellas. Por otra parte, muchas de las bases de datos de las empresas no están especialmente diseñadas para captarla, y los datos del balance tampoco la captan. Por lo tanto, se está construyendo una literatura empírica basada en estudios de casos, informes sectoriales o recopilación de datos ad hoc. Evidentemente, estos enfoques pueden proporcionar valiosas percepciones, pero no pueden dar una idea de la extensión general ni de las pautas de este fenómeno.

En este estudio utilizamos la base de datos del European Reshoring Monitor (ERM) que capta este fenómeno. De esta manera, presentamos por primera vez la extensión y las características de estos cambios de ubicación de segunda etapa que desarrollan las empresas. Los resultados revelarán el atractivo (y/o sus cambios) de algunos países para acoger actividades intermedias, así como el tipo de estrategias que las empresas de cada país están desarrollando. Además, investigar acerca de los procesos de retorno hacia la Unión Europea de las empresas (en concreto: producción, abastecimiento, investigación y desarrollo, servicios), puede representar un factor desencadenante clave para renovar la industria manufacturera de la UE en los próximos años y restablecer la UE como un lugar de excelencia mundial en materia de fabricación (European Reshoring Monitor 2018).

Desde el punto de vista metodológico, el análisis de red, aplicado ha supuesto una herramienta fundamental. Por un lado, el dinamismo que suponen las estrategias de offshoring y reshoring con desplazamientos espaciales desde unos países a otros. Por otro lado, el poder de visualización de esta metodología

El trabajo está estructurado de la siguiente manera. En la sección dos se resumen algunas de las publicaciones sobre el traslado de las actividades de las empresas. En la tercera sección se presentan las características de la base de datos, destacando sus rasgos especiales. En la cuarta sección se presenta la metodología de las redes que ilustrará qué países están vinculados, y cómo, en las decisiones que adopten las empresas en relación con la localización de sus actividades. En la quinta sección se presentan los resultados del análisis y en la última sección se resumen las principales conclusiones.

\section{Relocalización: Principales conceptos}

Para aumentar la eficiencia de la empresa, la localización de la producción es una de las estrategias clave. Una amplia literatura se ha centrado en qué cuestiones (desde la dotación de inputs, su coste, los costes de transporte, etc.) los determinan. A lo largo de las últimas décadas del siglo $\mathrm{XX}$, gracias a la digitalización y a las nuevas estructuras organizativas, surgió la posibilidad de que se produjeran más procesos de fragmentación del proceso de producción $y$, con ello, desapareció la co-localización de cada una de ellas. Junto a ello, surgió un replanteamiento de los límites de la empresa, llamando a las empresas a centrarse en aquellas actividades en las que sus competencias básicas les proporcionan una ventaja competitiva y buscando proveedores externos que les proporcionen cualquier input (bien o servicio) que puedan necesitar. La combinación de estas dos cuestiones llevó a las empresas a considerar conjuntamente la provisión interna, así como el lugar donde se llevaría a cabo la producción. Para el resultado de esta doble estrategia se utilizaron términos diferentes, y a veces no uniformes (como externalización, deslocalización...). Un resumen que se utilizó se presenta en la tabla 1 . 
Tabla 1 Tipos de offshoring y outsourcing.

Fuente: UNCTAD, 2004

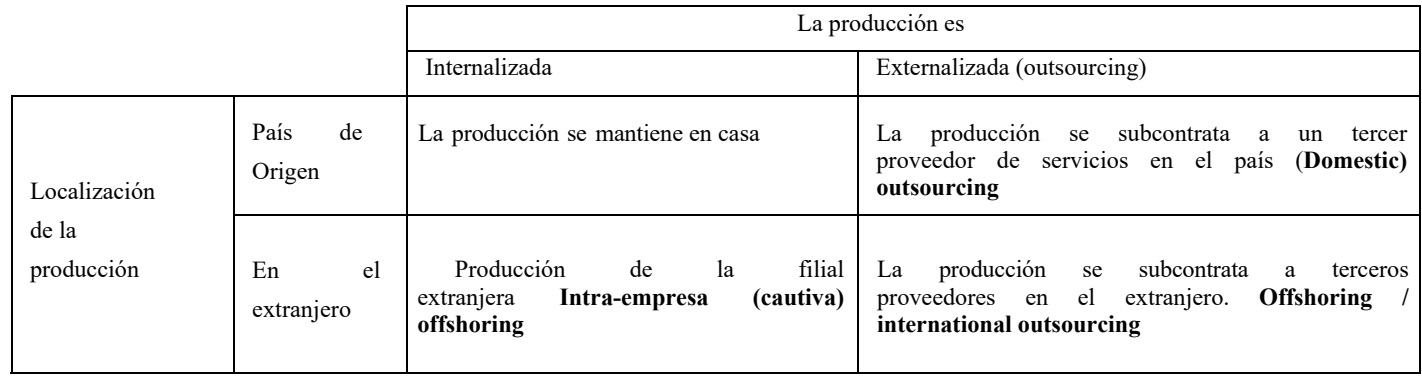

Tate y Bals (2017) resumen otros enfoques, como Foerstl y otros (2016), que amplía este marco para incluir explícitamente una diferenciación más detallada de la ubicación geográfica de las actividades. Distingue los destinos cercanos al país de origen (utilizando el término "nearshoring") de los lejanos; además, se incluye la distinción entre "on-site" y "offsite" en el caso de la prestación de servicios desde el país de origen (véase Jahns y otros, 2006), aunque parece ser pertinente sólo en el caso de las tareas de servicios y no tanto en la fabricación. Mientras tanto, otros autores (véase la referencia en Jahns y otros 2006) destacan la importancia de distinguir entre los modos de suministro intermedio en los que la empresa usuaria participa en la propiedad de la organización que suministra esos insumos con otros agentes, destacando un modo de suministro híbrido entre las alternativas de "fabricación" y "compra" como en el caso de las empresas mixtas.

A lo largo de los últimos años del siglo XX, pero especialmente en las dos primeras décadas del siglo XXI, comenzaron a producirse algunos casos de anulación de esos cambios. Las empresas que anteriormente habían desplazado su producción a terceros países (en algunos casos junto con un cambio en la decisión de internacionalización, en otros no) cambiaron de ubicación, ya sea a otro país extranjero o de vuelta al país de origen (de nuevo, en algunos casos cambiando el modo de suministro interno/externo de las actividades o no). Los casos más llamativos fueron los de producción devuelta al país de origen, dada la gran preocupación que había surgido en algunas economías avanzadas por la pérdida de puestos de trabajo (pero también por la recaudación de impuestos o la capacidad de innovación de las actividades que permanecían en el país de origen). Es más, algunos países trataron de alentar ese retorno mediante políticas diferentes.

Como ocurrió cuando la primera ola de deslocalizaciones comenzó a extenderse por las economías avanzadas, descubrimos que las empresas aplicaban diferentes tipos de estrategias, pero la falta de una terminología uniforme estaba presente tanto en los textos académicos como en los profesionales. Pudimos encontrar términos como reubicación, re-shoring, back-shoring, para el fenómeno que básicamente estaban deshaciendo un cambio anterior de la primera fila de la tabla 1 a la segunda.

Además, se observaron algunos cambios de localización adicionales en los casos en que las empresas no regresaban a sus países de origen las actividades que anteriormente se habían alejado de ellos. Las empresas trasladaron su producción a otros países lejanos para aprovechar las ventajas (puede ser porque el primer destino había perdido su atractivo, puede ser porque existían nuevas oportunidades, puede ser porque la empresa había cambiado su cadena de producción) pero también a países vecinos desde el lugar de origen de la empresa.

Todo ello provocó un desorden relativamente importante en la terminología que, paso a paso, se iba fijando dada su importancia para fomentar los estudios académicos posteriores (Foerst et al 2016).

En estos traslados de segunda etapa que pueden calificarse de "reorientación" (dejando de lado la cuestión del modo de provisión, ya que no es el tema de este documento), encontramos tres opciones diferentes:

- Backshoring: Empresas que después de trasladar su producción a un tercer país (ya sea en los locales de la empresa o mediante un contrato con un tercer agente) deciden volver a trasladarla con destino al país de origen. Este es el caso de "regreso a casa" de una, digamos, empresa francesa que trasladó su producción a China, pero decide volver a Francia de nuevo.

- Nearshoring: Empresas que después de trasladar su producción a un tercer país, deciden hacer un nuevo cambio de ubicación cuyo destino es un país vecino de la inicial que era el país de origen de la empresa inicialmente. Desde una perspectiva general, puede ser difícil delimitar lo que es un país vecino, pero cuando el análisis se desarrolla a los casos observados, suele estar claro (por ejemplo, empresas que se trasladaron de un país europeo a China y luego se trasladan de nuevo a otro país europeo o a Marruecos, Turquía...).

- $\quad$ Mayor offshoring: Empresas que después de trasladar su producción a un tercer país, deciden trasladarla de nuevo a un nuevo tercer país en el que no existe ningún 
tipo de proximidad con el país de origen. Este sería el caso de una empresa alemana que ha trasladado su producción a China y decide volver a trasladarla, pero con destino a la India, por ejemplo.

Un punto importante para destacar aquí es que el país es la unidad geográfica de análisis, se trata de, por ejemplo, una empresa alemana de Baviera que después de deslocalizar en China decide volver a Alemania, pero a otra zona (digamos Sajonia) y será considerada como de retroceso.

Cabe señalar que esta terminología no se utiliza de manera uniforme y que algunos autores llaman relocalización sólo a los casos de relocalización, así como otros que no consideran como parte de esta segunda etapa de relocalización a las empresas que hacen "further offshoring".

\section{Base de datos}

Este trabajo utiliza la base de datos del ERM que reúne información sobre casos individuales de deslocalización de diversas fuentes (medios de comunicación, prensa especializada, literatura científica, literatura para profesionales) y la organiza en una base de datos en línea de acceso seguro y actualizada periódicamente. El organismo encargado de publicar los informes y la actualización y gestión de la información es el Eurofound (European Foundation for the improvement of Living and Working Conditions) de la Comisión Europea. El criterio temporal es plurianual (2015-2018) para 250 empresas analizadas.

Como indica el ERM la información que proporciona se basa en:

1) Empresas que se deslocalizan a su país de origen (dentro de la UE) actividades de la cadena de valor que anteriormente se habían deslocalizado a otro país (por ejemplo, la fabricación de una empresa alemana que anteriormente se había deslocalizado a China o a Francia y que ahora regresa a Alemania).

2) Empresas que se deslocalizan a cualquier país de la UE con actividades de la cadena de valor que anteriormente se habían deslocalizado a un país no perteneciente a la UE (por ejemplo, la fabricación de una empresa alemana que anteriormente se había deslocalizado a China y ahora regresa a Italia).

\section{Metodología}

La metodología utilizada ha sido el análisis de redes, para determinar las relaciones de las empresas y sus vinculaciones entre los países de origen, y sus estrategias de Offshoring,
Reshoring y vinculaciones sectoriales. Se utilizan dos tipos de software; Gephi por su potencia visual. En este sentido, se utilizarán los algoritmos de Force Atlas para la definición de la red y de Louvain para la optimización del modularidad) y de cálculo e yEd de forma complementaria, por su sencillez. En relación con el concepto de modularidad, se considera comunidad a las relaciones descubiertas empíricamente entre los diferentes elementos de la red de acuerdo con Scott (1991). La metodología de análisis de red ha sido utilizada en ámbito empresarial por Borgatti et al (2009); Graf (2011) o Molina-Morales et al (2012) entre otros.

El objetivo se centra en conocer la posición y relevancia de los países (nodos) en la red o estructura, en relación con las decisiones estratégicas tomadas por las empresas. En particular la dinámica de flujo por los procesos de des(re) localización. Como fue mencionado, se analizarán, las estrategias de Offshoring (origen-destino1), Reshoring (destino1-destino2), y re-Reshoring (Reshoring-origen o destino3).

Lo habitual es utilizar los indicadores de centralidad y capacidad de intermediación, es decir, el grado (degree), la cercanía (closeness) y la intermediación (betweenness). Sin embargo, las medidas de entrada (in-degree) y salida (out-degree) con pesos ofrecen un mayor poder explicativo, que las mencionadas. Estas medidas suponen considerar el flujo de entrada/salida (empresas) en los nodos (países). Las correlaciones entre todas las medidas de centralidad mencionadas tienden a ser elevadas, en función del tipo de la red (densidad, simetría, etc.). Por ejemplo, en el caso de las medidas de entrada y salida, su grado de correlación sería de 1 , cuando la red fuera simétrica y por tanto el volumen de entradas y salidas fuera idéntico. Autores como Borgatti y Everett (1999); Costenbader y Valente (2003); Valente et al. (2008) ofrecen un interesante análisis sobre la funcionalidad y correlación de las medidas de centralidad en el análisis de redes.

En el caso de transición de las estrategias de re-reshoring, se ha optado por la utilización del software yEd, dado que muestra de forma más evidente la dinámica seguida por las empresas y sus destinos. Este software, muestra para cualquier medida valores entre 0 y 1 , indicando su relevancia en el indicador elegido en función de su proximidad a 1 .

\section{Resultados}

Para enmarcar el análisis de las estrategias de offshoring y proporcionar una primera visión de la base de datos, la tabla 2 muestra los valores de los diez países que ocupan una mayor centralidad y mayores niveles de relaciones en función del indicador. 
Tabla 2 Valores de los indicadores elegidos de los principales nodos/países en estrategias origenoffshoring.

Fuente: ERM y elaboración propia

\begin{tabular}{lcccc}
\hline País & $\begin{array}{c}\text { Grado entrada } \\
\text { con pesos }\end{array}$ & $\begin{array}{c}\text { Grado salida } \\
\text { con pesos }\end{array}$ & $\begin{array}{c}\text { Grado } \\
\text { con pesos }\end{array}$ & Comunidades \\
\hline China & 76 & 0 & 76 & 2 \\
India & 15 & 0 & 15 & 1 \\
Polonia & 15 & 6 & 21 & 2 \\
Alemania & 14 & 17 & 31 & 0 \\
Suecia & 9 & 17 & 26 & 1 \\
Reino Unido & 9 & 40 & 49 & 0 \\
Estados Unidos & 7 & 2 & 9 & 2 \\
Francia & 6 & 34 & 40 & 3 \\
Países Bajos & 6 & 4 & 43 & 3 \\
Italia & 6 & 37 & 43 & 2 \\
\hline
\end{tabular}

Como muestra la tabla 2, ordenada según los valores de entrada ponderada (teniendo en cuenta las interacciones nodales), China ocupa el primer lugar, es decir es el país de mayor intensidad receptora en empresas que realizan offshoring seguido por India, Polonia, Alemania, etcétera. Sin embargo, atendiendo al indicador de salida ponderado, no se producen estrategias de offshoring (out-degree), considerando orígenes a China o India.

La importancia de posicionamiento local en la red, la proporciona el indicador de grado con pesos. China es el que ocupa la posición más relevante, seguida por Inglaterra. Por último, la columna Comunidades, muestra la existencia de 4 comunidades o países que están fuertemente conectados.
Figura 1 proporciona un diagrama de red de la decisión de deslocalización offshoring, utilizando el grado de salida. Como puede apreciarse en la figura, China como ya ha sido mencionado es el país receptor más importante de empresas, cuya densidad de aristas (in-degree) se muestran por el grosor de estas. Le siguen a mucha distancia Alemania, Francia, Italia, India, Polonia o Suecia respectivamente. Los colores de nodos y flechas capturan las comunidades o interacciones entre países o empresas. La representatividad de la comunidad en color magenta muestra un importante dinamismo de interacciones, frente al resto.

Figura 1 Flujo de las

Estrategias de offshoring desde el origen 2015-2018.

Fuente: ERM y elaboración propia

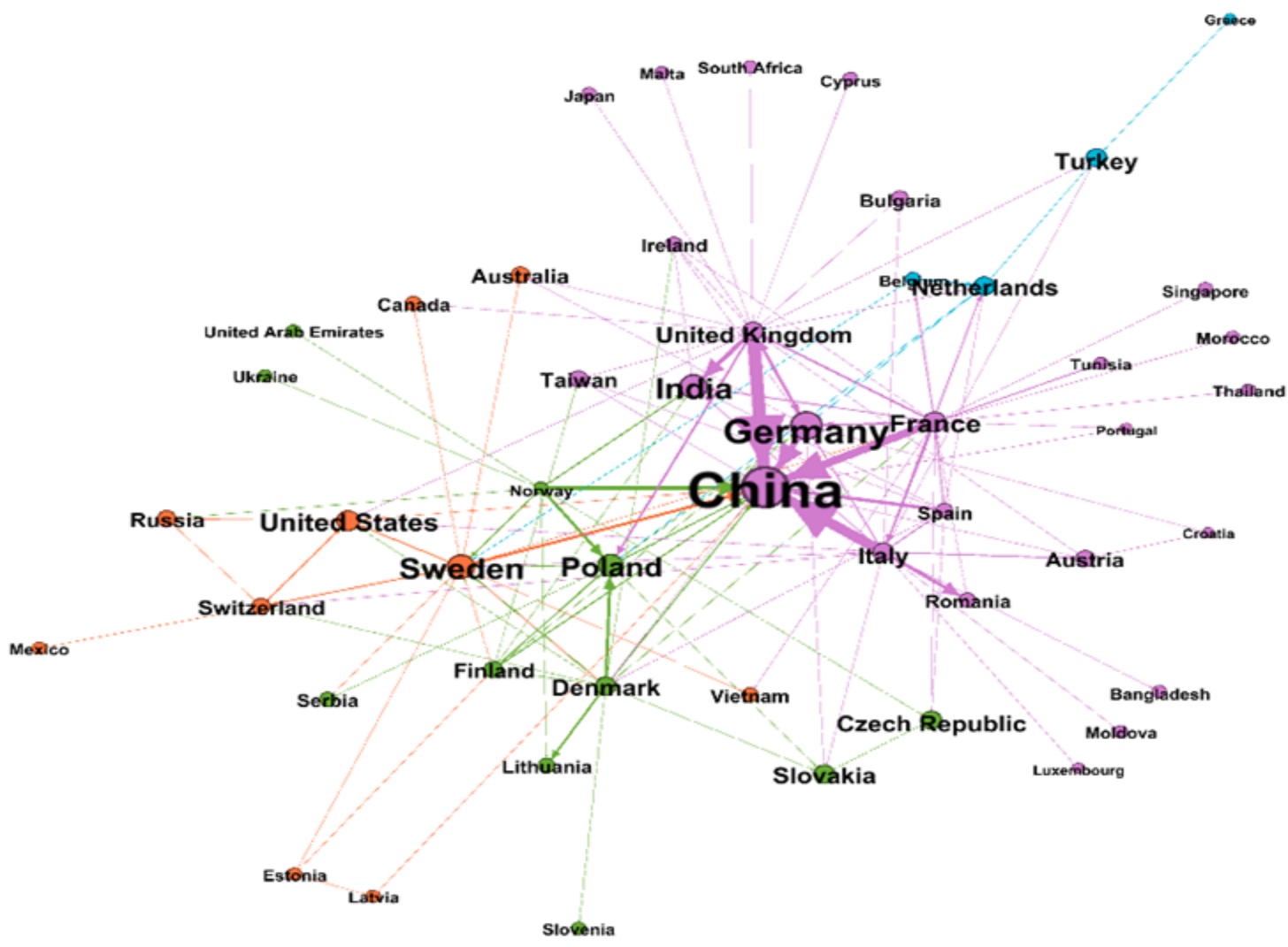


Como es bien sabido, a lo largo de este período China se convirtió en un imán para muchas actividades económicas, sobre todo manufactureras, gracias a sus bajos costos laborales, su pertenencia a la Organización Mundial del Comercio y a políticas específicas para atraer a los inversores extranjeros que también podían beneficiarse de una enorme comercialización nacional. Como resultado de esos cambios, muchas empresas de economías avanzadas trasladaron su producción a China, ya sea mediante contratos en condiciones de igualdad con las empresas locales o invirtiendo en instalaciones en ese país. Los resultados de los análisis presentados en la figura 1 confirman esta tendencia. Como se puede observar, China surge como el principal protagonista de las importaciones en este fenómeno, según los datos utilizados. Los orígenes son las principales economías europeas (Reino Unido, Alemania, Italia, Francia). El caso de la India también es interesante, es el destino de muchas decisiones de deslocalización, pero principalmente del Reino Unido. Entre los países europeos, encontramos que algunos de Europa Central y del Este emergen también como destino de las decisiones de deslocalización, siendo Polonia el líder de este grupo.

Atendiendo a los sectores donde se producen los procesos de offshoring, las figuras 2.1 y 2.2 muestran la densidad o representación de los sectores, C26, C27, C30, C32 o C14
Figura 2.1 Sectores

implicados en el proceso de offshoring (zoom).

Fuente: ERM y elaboración propia

Figura 2.2 Zoom de sectores implicados en el proceso de offshoring Fuente: ERM y elaboración propia
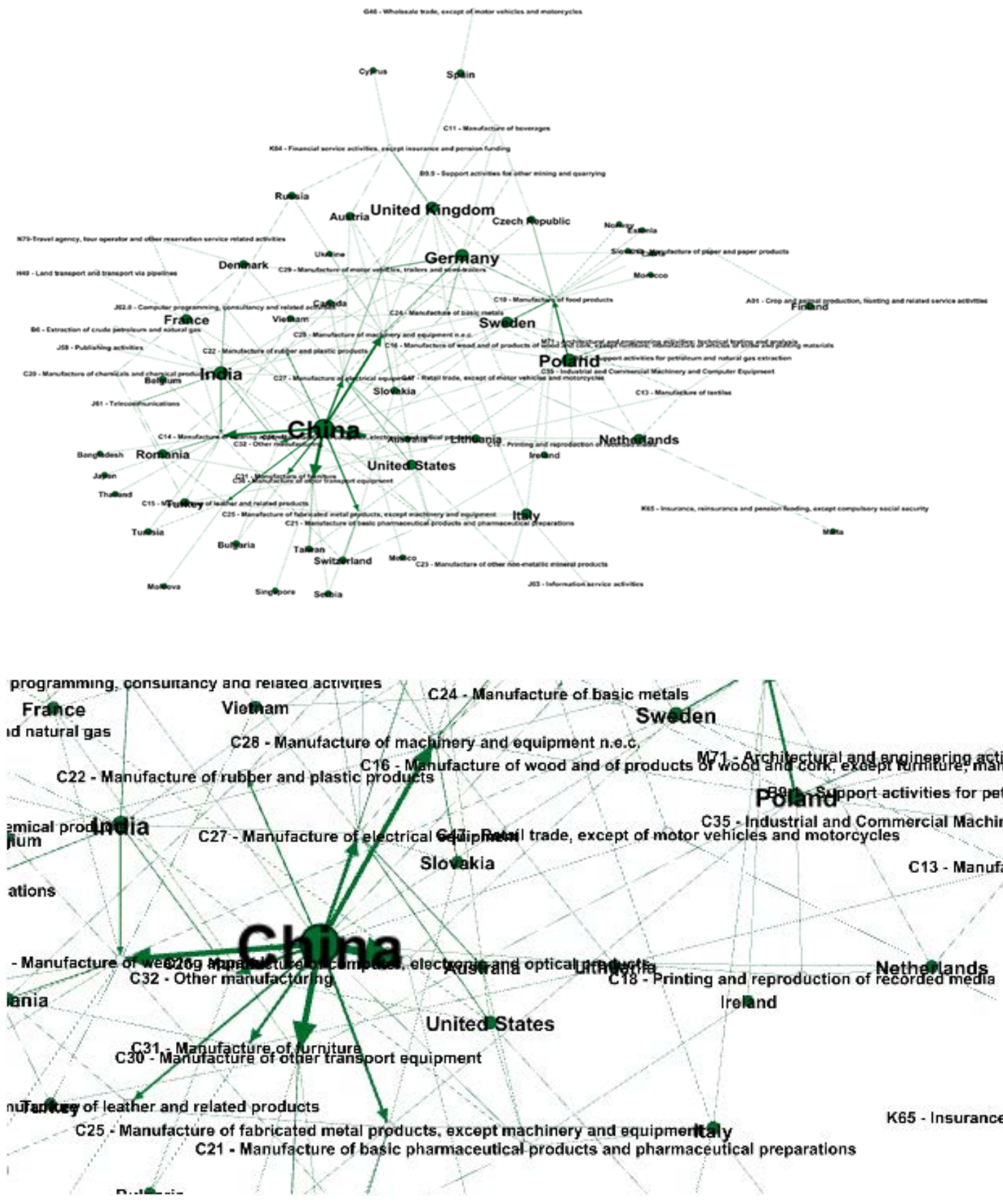
Uno de los objetivos principales es analizar cuáles son los destinos de las decisiones de reorientación, es decir, hacia dónde se trasladaron las actividades. Como podemos ver en la figura 3, China, es el destino central de la deslocalización, se convierte lógicamente en el centro del fenómeno de reorientación. Como se observa, la mayoría de los casos de reorientación fueron empresas que se desplazaron hacia economías avanzadas (el Reino Unido, Francia e Italia son los más importantes, aunque Suecia y Noruega también se convierten en destinos importantes para las actividades que salen de China). Una mirada más atenta revela que Polonia es un destino importante, mientras que otros países distantes como la India no lo son. Así pues, podemos encontrar tres tipos de movimientos:
1) De China a las economías avanzadas, sobre todo de reorientación, se trata de empresas que en el pasado trasladaron su producción a China en busca de ventajas de costes y que por cualquier motivo decidieron reorientar (no tan grandes ventajas de costes como se preveía, costes más altos que los esperados de gestionar una producción geográfica y culturalmente distante).

2) De China a países como Polonia, donde la producción se sustituye a países más cercanos al mercado europeo y a la sede de la empresa, lo que facilita y abarata su gestión.

3) De China a otros países de bajo coste como la India, probablemente motivado por el aumento de costes que ha experimentado China y/o la necesidad de buscar ventajas de costes adicionales.

Figura 3 Flujo de las

Estrategias de Reshoring

2015-2018.

Fuente: ERM y elaboración

propia

Es interesante observar que el fenómeno de reorientación no se limita a las empresas que salen de China y que también se dan casos entre las economías avanzadas, probablemente debido a la reorganización interna de las empresas. Incluso con estos casos, podemos observar cómo China es el elemento central del fenómeno de reorientación.

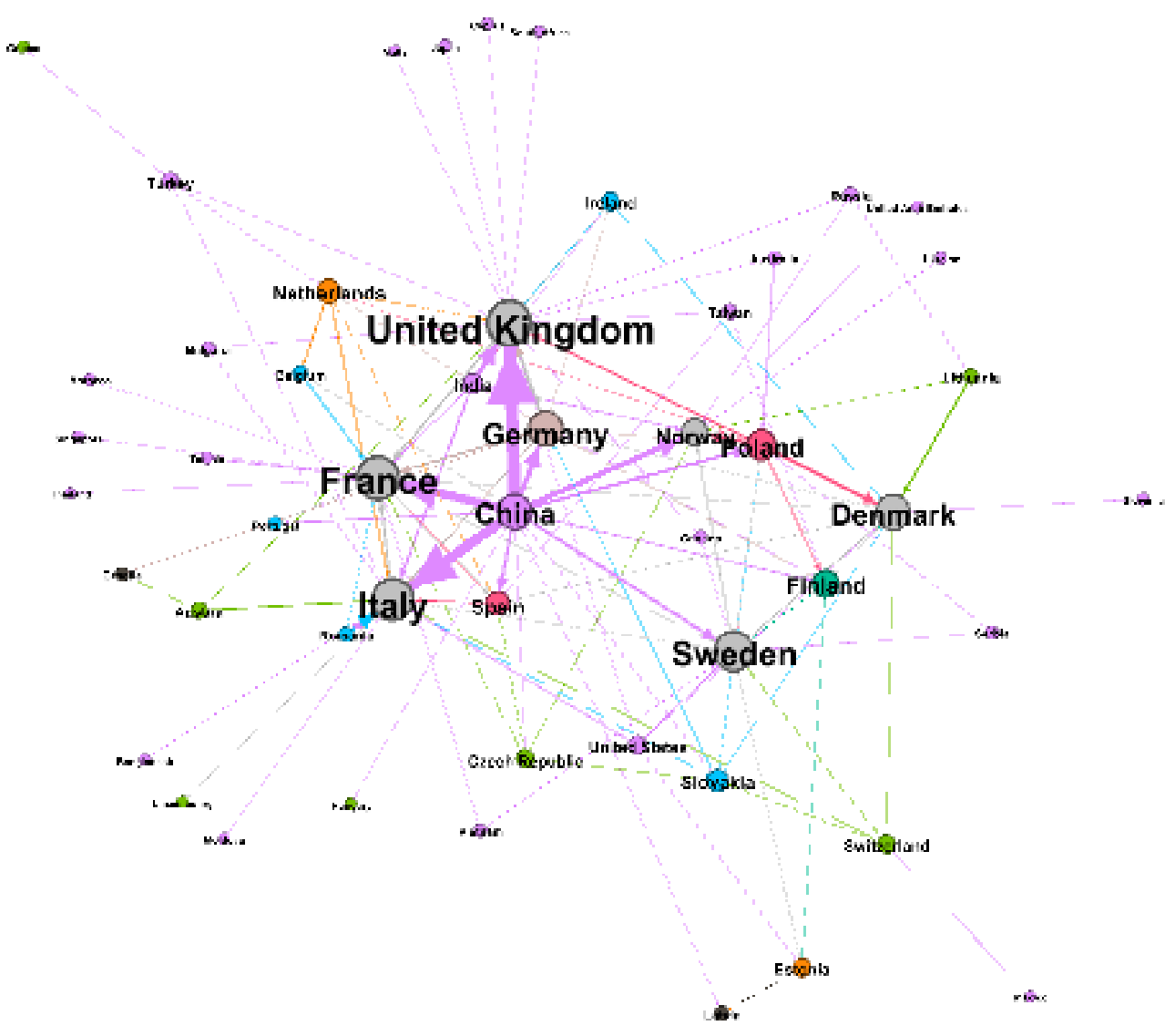

La figura 4 muestra el número de empresas que se deslocalizan y regresan a su país de origen, estos son los casos de "backshoring". Con esta información, es posible saber con qué intensidad se produce el back-shoring en cada país. Como podemos ver Francia, Reino Unido e Italia es donde la estrategia de "backshoring" es más común, aunque incluso en casos como Noruega, Dinamarca, Suecia, Polonia, etc. está sucediendo. 
Figura 4 Flujo de las Estrategias de Reshoring 2015-2018.

Fuente: ERM y elaboración propia

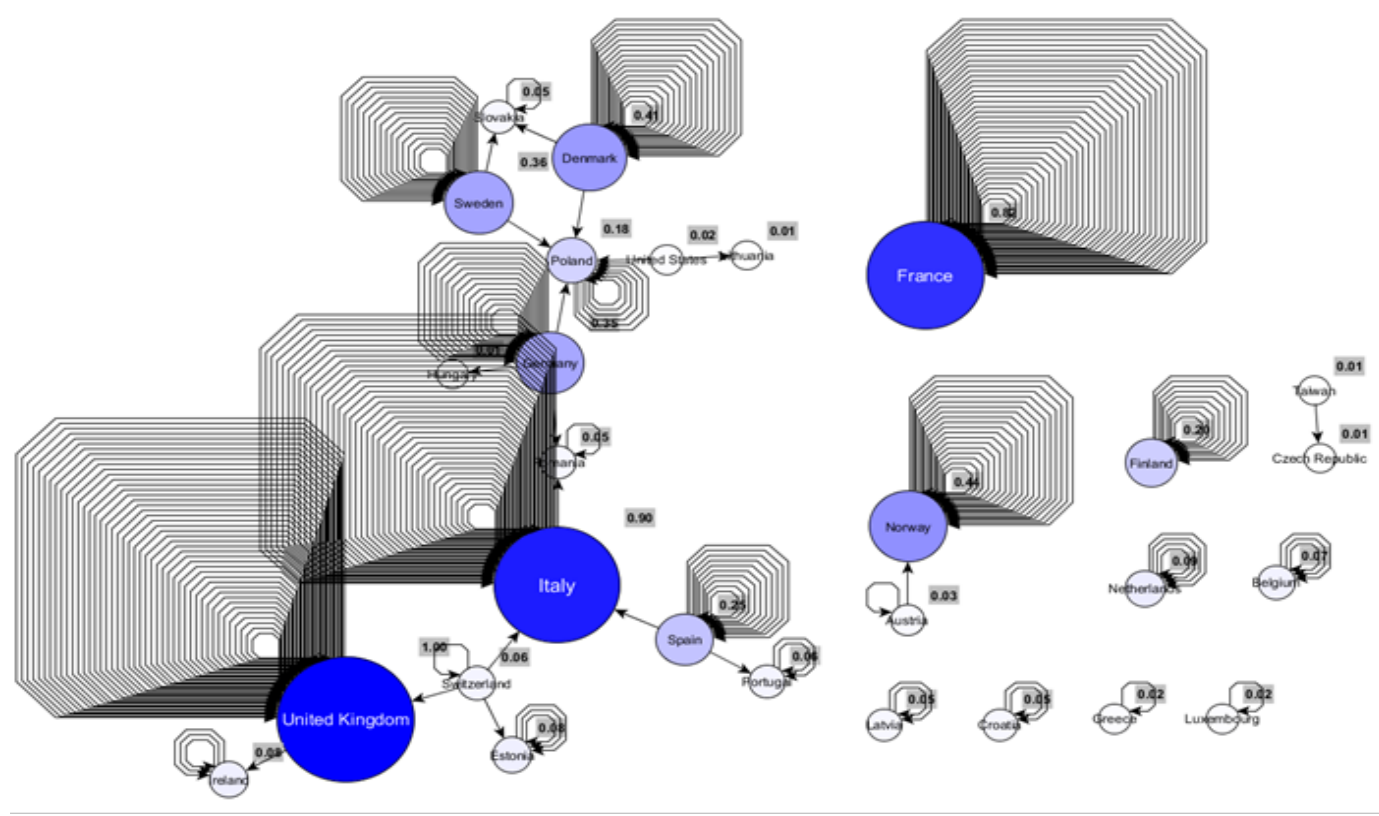

\section{6.- Conclusiones}

Para las empresas, la localización de su actividad es una de las estrategias-clave para su competitividad en los mercados. La ubicación no sólo determina los costes de transporte (tanto del producto final como de los bienes intermedios y materias primas), sino los costes de producción (derivados de las diferencias en factores clave como la mano de obra o la energía, por ejemplo) o la regulación a la que puedan estar sometidas de cara a llevar a cabo su producción. La irrupción de factores externos a la empresa (como la digitalización, la reducción de barreras al comercio internacional, la aparición de nuevas posibilidades de localización en el mundo, etc.) modifica la optimalidad de una estrategia de localizaciones.

Uno de estos cambios lo encontramos en las dos últimas décadas del siglo XX (y primera del XXI) cuando distintos factores suponen la irrupción de un conjunto muy importante de países en la economía global. Desde las empresas, este cambio llevó a una importante ola de deslocalizaciones, por la vía del offshoring, que permitieron la obtención de productos a menor coste, promovieron el crecimiento económico en los países en los que se localizaba la producción, pero también suponía un riesgo para el tejido producto en los países que abandonaban.

En los últimos diez años, se observan diferentes casos de empresas que han deshecho este camino, algunas de ellas para buscar nuevas ubicaciones para toda (o parte) de su cadena de valor en países con nuevas ventajas competitivas y otras para volver al país de origen de la empresa. La literatura económica ha abordado este fenómeno, tanto por el interés de este cambio como por las consecuencias sobre los países. Como se ha señalado en el trabajo, las aproximaciones han tenido una perspectiva muy variada, pero no se ha abordado una visión geográfica general en gran medida por la dificultad de obtener datos que permitiesen hacerlo.

En este trabajo, aprovechando la información proporcionada por el ERM, cuyas características y limitaciones se han descrito, se aborda por primera vez el papel que cada país tiene dentro de este fenómeno.

La primera conclusión es la importancia de China como destino de la ola inicial de offshoring, resultado que concuerda con la importante atracción de flujos de IDE de este país (que fue más allá de abastecer un gran mercado con una potencialidad de crecimiento muy superior al de los países desarrollados) y de comercio internacional. Debe recordarse que, al margen del crecimiento interno chino, las exportaciones de este país pasaron del entorno del $2 \%$ de su economía a más del $35 \%$ en los años anteriores a la Gran Recesión. Los resultados de este trabajo muestran que no sólo países emergentes como China fueron el destino de la oleada de offshoring, sino que también países avanzados como Alemania o Italia fueron el destino de estos cambios en la localización de la producción.

La oleada de segundos cambios en la localización (algunos hacia países en los que explotar ventajas adicionales de costes, pero otros de retorno a economías avanzadas o al país de origen) tiene, lógicamente en China su epicentro. El destino de estos cambios es, mayoritariamente el de países avanzados (Reino Unido, Francia, Italia, países escandinavos) ocupando un papel minoritario India, países africanos o latinoamericanos. Es decir, la segunda oleada de relocalización no ha tenido como destino la búsqueda de "otras Chinas" donde poder explotar ventajas adicionales 
de coste, sino el retorno a países avanzados motivada por una pluralidad de factores que la literatura ha puesto de manifiesto. Un caso para destacar es el de Polonia, que ocupa una posición importante en la red de relocalizaciones al ser destino importante de empresas que previamente habían hecho offshoring. La razón de la relevancia de este país se encuentra en que teniendo proximidad geográfica a las economías más avanzadas y muchas de las garantías de estas (acceso al mercado derivado de su pertenencia a la UE, seguridad jurídica, etc.), mantiene un diferencial de costes importante con países centrales de la UE como Alemania, Holanda o Francia, por ejemplo.

Como ya se ha indicado, en esta segunda oleada de relocalizaciones, las economías avanzadas ocupan una posición dominante en la red. También se ha estudiado si realmente se trata de casos de backshoring, esto es, si la empresa vuelve al país de origen o si se ubica en otro país avanzado. Los resultados han puesto de manifiesto que el backshoring es más común entre empresas francesas, británicas e italianas. La literatura que ha estudiado cada país ha ido señalando la importancia de factores distintos en cada uno de ellos, lo que puede explicar la heterogeneidad de los resultados alcanzados.

Los resultados de este trabajo plantean líneas de investigación interesantes para abordar el fenómeno que van desde las razones para estos casos y si puede plantearse un marco común más allá de los ya discutidos (como Benstead y otros 2007) o las consecuencias para las empresas en términos de competitividad y rentabilidad de estas estrategias o su impacto sobre la economía local.

\section{Agradecimientos:}

Los autores agradecen las sugerencias realizadas por José Luis Molina (Universidad Autónoma de Barcelona) en lo referente a la metodología utilizada. Cualquier error es responsabilidad de los autores de este trabajo.

\section{Referencias bibliográficas}

AMITI, M. AND WEI, S-J. (2009), "Service Offshoring and Productivity: Evidence from the US", The World Economy, 32, pp. 203-220

BENSTEAD, A.V., STEVENSON, M. AND HENDRY, L.C. (2017), "Why and how do firms reshore? A contingency-based conceptual framework", Operations Management Research, 10, pp. 85-113.
BOEHM, C., FLAAEN, A. AND PANDALAI-NAYAR, N. (2017), "Multinationals, Offshoring and the Decline of U.S. Manufacturing”, NBER Working Paper No. 25824

BORGATTI, S., MEHRA, A., BRASS, D., LABIANCA, G., 2009. Network. Analysis in the Social Sciences. Science 323, 892-895.

COSTENBADER, E. AND VALENTE, T. (2003). The stability of centrality measures when networks are sampled. Social Networks. 25: 283-307

MOLINA-MORALES, F.X, CAPÓ-VICEDO, J., TOMÁS-MIQUEL, J.V. EXPÓSITO-LANGA, M. (2012). Análisis de las redes de negocio y de conocimiento en un distrito industrial. Una aplicación al distrito industrial textil valenciano. Cuadernos de Economía y Dirección de la Empresa 15, 94-102

CARLUCCIO, J., CUÑAT, A., FADINGER, H. AND FONS-ROSEN, C. (2019), "Offshoring and skill-upgrading in French manufacturing”, Journal of International Economics, 118, pp. 138-159

ELLRAM, L, TATE, W. AND PETERSEN, K. (2013). Offshoring and Reshoring: An Update on the Manufacturing Location Decision, Journal of Supply Chain Management, 49, pp. 14-22.

FOERSTL, K., KIRCHOFF, J.F., \& BALS, L. (2016). "Reshoring and Insourcing: Drivers and Future Research Directions", International Journal of Physical Distribution and Logistics Management, 46 (5), pp. 492-515.

GARNER, C. (2004), "Offshoring in the Service Sector: Economic Impact and Policy Issues", Economic Review - Federal Reserve Bank of Kansas City; 89, pp. 5-39.

GRAF, H., 2011. Gatekeepers in regional networks of innovators. Cambridge Journal of Economics, 35, pp. 173-198.

HEAD K. MAYER, T. AND RIES, J. (2009), "How remote is the offshoring threat?" European Economic Review, 53, pp. 429-444.

JAHNS, C., HARTMANN, E., BALS, L. (2006). "Offshoring: Dimensions and diffusion of a new business concept", Journal of Purchasing \& Supply Management, 12, pp. 218-231. 
KINKEL, S. AND ZANKER, C. (2013), "New patterns of German production relocation and backshoring activities after the global economic crisis", Euro MA Conference, Dublin, Ireland, pp. 7-12.

KINKEL, S., LAY, G. AND MALOCA, S. (2007), "Development, motives and employment effects of manufacturing offshoring of German SMEs", International Journal Entrepreneurship and Small Business, 3 (4), pp. 256-276.

MARTÍNEZ-MORA, C. AND MERINO, F. (2014), "Offshoring in the Spanish footwear industry: a return journey?" Journal of Purchasing and Supply Management, 4 (20), pp. 225-237.

SCOTT, J. (1991). Social Network Analysis. A Handbook. London: (Sage).
TATE, W. (2014), "Offshoring and reshoring: U.S. insights and research challenges", Journal of Purchasing \& Supply Management, 20, pp. 66-68.

TATE, W. AND BALS L. (2017)," Outsourcing/offshoring insights: going beyond reshoring to rightshoring ", International Journal of Physical Distribution \& Logistics Management, 47, pp. 106 - 113

TATE, W.L., BALS, L. (2017). “Outsourcing/offshoring insights: going beyond reshoring to rightshoring", International Journal of Physical Distribution \& Logistics Management, 47(2/3), pp. 106-113.

UNCTAD (2013), "World Investment Report”, United Nations, Geneva, New York.

VALENTE, T.W., CORONGES, K., LAKON, C. AND COSTENBADER, E. (2008). How Correlated Are Network Centrality Measures? National of Health Institute Connect (Tor) 1, 28(1), pp. 16-26. 